\title{
Primary diffuse large B-cell lymphoma of the bone mimicking osteomyelitis
}

\author{
abbas mofidi ${ }^{1}$, Mohsen Esfandbod ${ }^{2}$, Ehsan Pendar ${ }^{2}$, Masoud Mortezazadeh ${ }^{2}$, and Alireza \\ hadizadeh $^{2}$ \\ ${ }^{1}$ Iran University of Medical Sciences \\ ${ }^{2}$ Tehran University of Medical Sciences
}

June 16, 2021

\begin{abstract}
In this article, we report a 34-year-old man who presented with progressive hip pain and osteolytic bone lesions . Primary workup included Core needle biopsies manifested as osteomyelitis; however as no sign of remission was observed, an open biopsy considered which revealed primary bone lymphoma.
\end{abstract}

\section{Introduction:}

Primary bone diffuse large B-cell lymphoma (PB-DLBCL) is a destructive primary extranodal lymphoma that accounts for $55.7 \%$ of all primary bone lymphomas. (1)

Patients are clinically presented with bone pain that is not relieved by rest, soft tissue swelling, palpable mass, pathologic fracture, joint contracture, cord compression, and systemic symptoms such as fever, unintentional weight loss, and night sweats. (2)

The common primary site of bone involvement is the axial skeleton rather than the appendicular skeleton (63 versus 37 percent ). (3)

The diagnosis of (PB-DLBCL) is based on both imaging and histopathologic findings. tissue samples can be taken either percutaneously or via open biopsy.

few primary bone lymphoma cases have been reported in the literature. These cases are usually misdiagnosed, particularly with osteomyelitis. the symptoms often persist for many months before the patient seeks appropriate medical care(4)

herein, we describe a 34-year-old young man with persistent bone pain whose core needle biopsies were consistent with osteomyelitis, however, his open biopsy revealed PB-DLBCL to be the underlying cause.

\section{Case Presentation:}

a 34-year-old man with no significant past medical history complaining of dull low back pain and nontraumatic progressive right hip pain which he had for 6 months was referred to our clinic. He described his pain as dull low back pain and he also localized his hip pain on the anterior part of the right hip, the pain also radiated to the buttock and groin, his pain also exacerbated with weight-bearing activities, and didn't relieve even with rest or pain killers such as morphine. the patient had been bedridden for a month as the pain had gotten excruciating. Meanwhile, the patient had no constitutional symptoms.

the examination revealed decreased range of motion on his right hip joint and tenderness over the anterior joint line, adjacent soft tissue to the right hip joint was also swollen and asymmetric compared to the left 
side, other physical exams were unremarkable.

in the previous workup and admission 6 months before referral, the Magnetic resonance imaging(MRI) had demonstrated diffuse abnormal high-intensity signals on $\mathrm{T} 2$ and proton density fat saturation in the right iliac and superior ramus of pubis was seen, this was accompanied by a high-intensity signal on the peripheral soft tissue along with periosteal reaction.

abnormal high-intensity signals in the medulla of the femur and sequestration in the iliac bone were also reported.

according to the imaging findings which were suggestive for infiltrative disorders. A core needle biopsy was obtained from the lesion in the right acetabulum the pathologic assessment reported unremarkable spongy bone with cellular marrow tissue and a benign lymphocytic aggregation.

these findings were suggestive of osteomyelitis, and subsequently, he was treated with antibiotics which turned out to be ineffective.

3 months after the first admission as the pain had progressed, he has admitted again for further evaluation and the imaging revealed progression of the lesion. ( Figure 1)

He became a candidate for another biopsy that showed inflamed fibro connective tissue and fibrin exudative substances which were compatible with osteomyelitis. He was treated again with broad-spectrum antibiotics for another 6 weeks.

As the patient's overall health and symptoms showed no sign of remission, he was referred to our clinic for further evaluation. He was admitted and samples were taken. laboratory results showed the following: white blood cell (WBC) count 5700/ $\mathrm{ll}$ (band+segment $59.0 \%$, mono $10.0 \%$, lymph $28.0 \%$ ); hemoglobin (Hgb) $11.5 \mathrm{~g} / \mathrm{dl}$; platelet count $(\mathrm{Plt}) 2.83 \times 10^{4} / \mu \mathrm{l}$; lactate dehydrogenase $(\mathrm{LDH}) 355 \mathrm{IU} / \mathrm{l}$ (normal range 150-500 U/L); AST 13 IU/L; ALT 16 IU/l; C-reactive protein (CRP) $25 \mathrm{mg} / \mathrm{dl}$; (ESR) $32 \mathrm{~mm} / \mathrm{h}$.

for evaluating the lesions a whole bone scan with TC-99m was performed which was suggestive for chronic arthritis and osteomyelitis in sacroiliac joint, right acetabular bone especially in the acetabular roof, and the right iliac crest, it was also suggestive for probable bone tumors. (Figure 2)

According to the clinical findings and the fact that previous core needle biopsies were inconclusive and unhelpful, we decided to perform an open biopsy to rule out primary bone malignancies.

The open biopsy of the lesion and the pathologic assessment suggested primary bone DLBCL.

Immunohistochemical results were as following LCA +, CD20+, Bcl-6+, Bcl 2 +, CD3- , MUM-1+, CD99

-, vimentin - , CD10 -, and Ki67+ (90\%), this was consistent with DLBCL, non-germinal center B-cell-like (non-GCB).(figure 3 )

using the Ann Arbor staging, the patient was staged at I EA level of the disease with no other distant metastasis.

The patient received his first cycle of chemotherapy with rituximab, cyclophosphamide, Adriamycin, vincristine, prednisone (R-CHOP), and concurrent intrathecal chemotherapy with Cytarabin, methotrexate, and hydrocortisone. meanwhile, He also became a candidate for radiotherapy (RT).

\section{Discussion:}

PB-DLBCL is rare extranodal lymphoma which accounts for 2 percent of all lymphomas in adult and the most common symptom is bone pain.

In this article, we report a 34-year-old man who presented progressive hip pain and osteolytic bone lesions. He was later diagnosed with primary diffuse large B-cell bone lymphoma, previous studies had also suggested that osteomyelitis unusually mimics PB-DLBCL (5-7) 
Several rationales led us to believe that the previous workup session was insufficient and another approach is needed to be taken, hence we decided to perform an open biopsy, one of the main reasons to do so was the fact that bone pain was progressive and unresponsive to the standard antibiotic regimen.

On the other hand Radiologic changes, such as osteolytic lesions, were progressive and despite regular treatment for osteomyelitis the lesions progressed and contained sequestrum as the disease spread. this pattern of progress was also observed between the two MRI scans that were taken in the 6 months. These changes include an extraosseous extension of the lesion that had led to asymmetric soft tissue swelling. These patterns of change are usually seen with neoplastic lesions rather than infectious ones.

In recent studies, it has been suggested that An ESR level of $60 \mathrm{~mm} / \mathrm{h}$ is an optimal cutoff level for suspected osteomyelitis. (74\% sensitive and 56\% specific), our patient had ESR levels below the threshold, thus osteomyelitis seemed unlikely. (8)

Other reasons such as lack of predisposing factors for osteomyelitis (i.e., Long term skin infections, Prosthetic joints, use of intravenous drugs, etc.) and abrupt beginning of the symptoms made our case less probable for osteomyelitis. Being safer and almost equally accurate it has been suggested by the literature that core needle biopsy is a more preferable method of obtaining bone tissue sample than open biopsy. it is also both less invasive and cost-effective. (9)

unfortunately, though in our case core needle biopsy couldn't help provide an accurate diagnosis.

we have come to conclude that the first two biopsies were taken from the rim of the lesion, thus making the sample redundant and inefficient. the result was only suggestive for non-specific lymphocyte aggregation. In such circumstances, open biopsy seems suitable for patients who are highly suspicious of bone tumors and core needle biopsy is inconclusive and non-diagnostic. (10)

\section{conclusion:}

In brief, our case illustrates the difficulties in distinguishing primary bone lymphoma from osteomyelitis as both have similar presentations, while the radiologic findings can overlap and mimic one another.

Our experience with this case emphasizes the fact that for an accurate diagnosis while the clinical manifestations and Para clinical findings are suggestive for malignancies and tumors, in this case, lymphoma, and the results from core needle biopsy are incoherent, open biopsy is to be considered.

\section{Declarations:}

\section{Funding: Not applicable}

2. Conflicts of interest/Competing interests: The authors declare that there is no conflict of interest to declare.

3. Ethics approval: In this study, no additional costs and procedures were imposed on the patient. We reported the standard treatment process of the patient retrograde. We maintained the patient's privacy, and her written consent was obtained.

4. Consent to participate: The patient has consented to the participation of this case report.

5. Consent for publication: The participant has consented to the publication of this case report.

6. Availability of data and material: The data that support the findings of this study are available from the corresponding author, $[\mathrm{MM}]$, upon reasonable request.

7. Code availability (Not applicable)

8. Authors' contributions: A.M. and M.M. contributed to data collection, writing, drafting of the manuscript, and critical appraisal of the manuscript. A.H,EP and M.E. contributed to scientific writing and final revision

\section{References :}


1. Zhang X, Zhu J, Song Y, Ping L, Zheng W. Clinical characterization and outcome of primary bone lymphoma: a retrospective study of 61 Chinese patients. Scientific Reports. 2016;6(1):28834.

2. Choi JY, Hahn JS, Suh CO, Yang WI. Primary lymphoma of bone-survival and prognosis. Korean J Intern Med. 2002;17(3):191-7.

3. Jawad MU, Schneiderbauer MM, Min ES, Cheung MC, Koniaris LG, Scully SP. Primary lymphoma of bone in adult patients. Cancer. 2010;116(4):871-9.

4. MIKA J, SCHLEICHER I, GERLACH U, ADLER C-P, UHL M, KNOELLER SM. Primary Bone Lymphomas Thought to Be Osteomyelitis Urgently Demand a Rapid Diagnosis in Bone Pathology. Anticancer Research. 2012;32(11):4905-12.

5. Hsieh TC, Kao CH, Yen KY, Sun SS. Osteomyelitis-mimicking primary bone lymphoma at hip prosthetic site. Clin Nucl Med. 2007;32(7):543-4.

6. Badam T, Devadoss S. Primary Bone Lymphoma - Mimicking as Osteomyelitis: An Unusual Presentation. Journal of Orthopedic Oncology. 2016;02.

7. Mika J, Schleicher I, Gerlach U, Adler C-P, Uhl M, Knoeller SM. Primary Bone Lymphomas Thought to Be Osteomyelitis Urgently Demand a Rapid Diagnosis in Bone Pathology. Anticancer Research. 2012;32(11):4905.

8. Lavery LA, Ahn J, Ryan EC, Bhavan K, Oz OK, La Fontaine J, et al. What are the Optimal Cutoff Values for ESR and CRP to Diagnose Osteomyelitis in Patients with Diabetes-related Foot Infections? Clin Orthop Relat Res. 2019;477(7):1594-602.

9. Pohlig F, Kirchhoff C, Lenze U, Schauwecker J, Burgkart R, Rechl H, et al. Percutaneous core needle biopsy versus open biopsy in diagnostics of bone and soft tissue sarcoma: a retrospective study. European Journal of Medical Research. 2012;17(1):29.

10. Majeed A, Chan O, Okolo O, Shponka V, Georgescu A, Persky D. Hodgkin Lymphoma Mimicking Osteomyelitis. Case Rep Oncol. 2017;10(2):542-7.

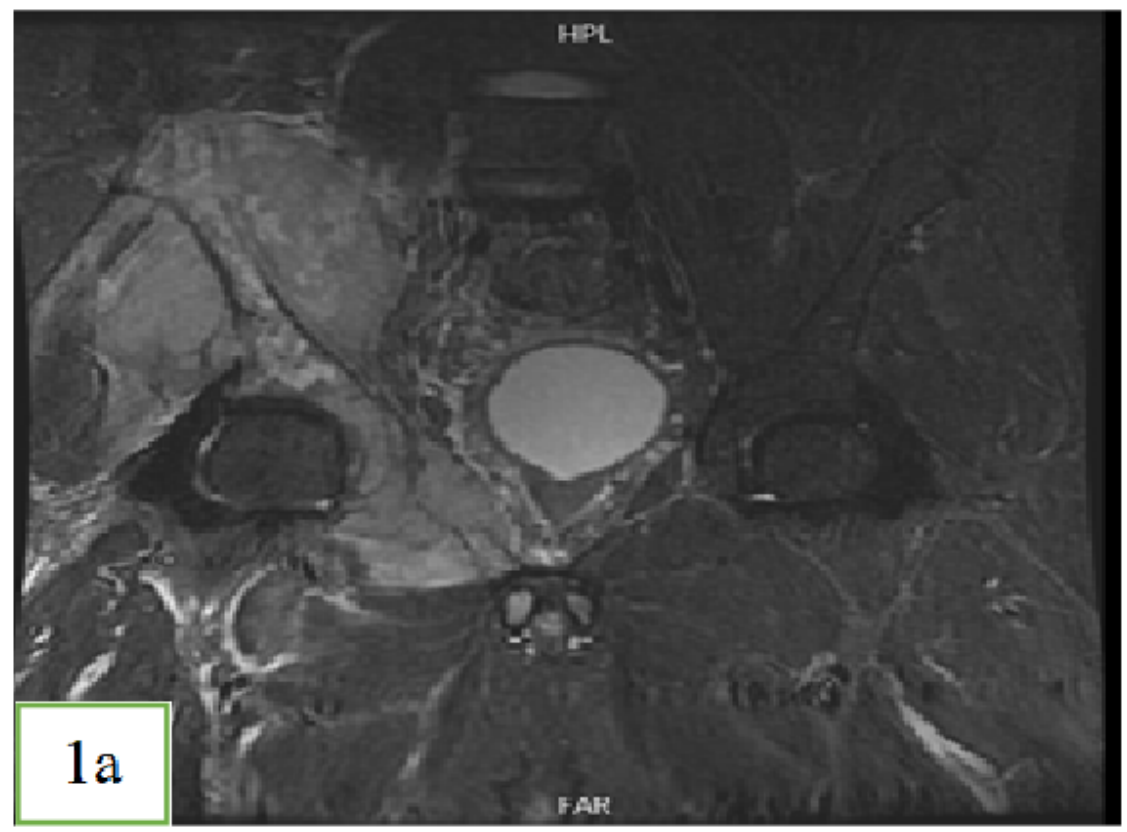



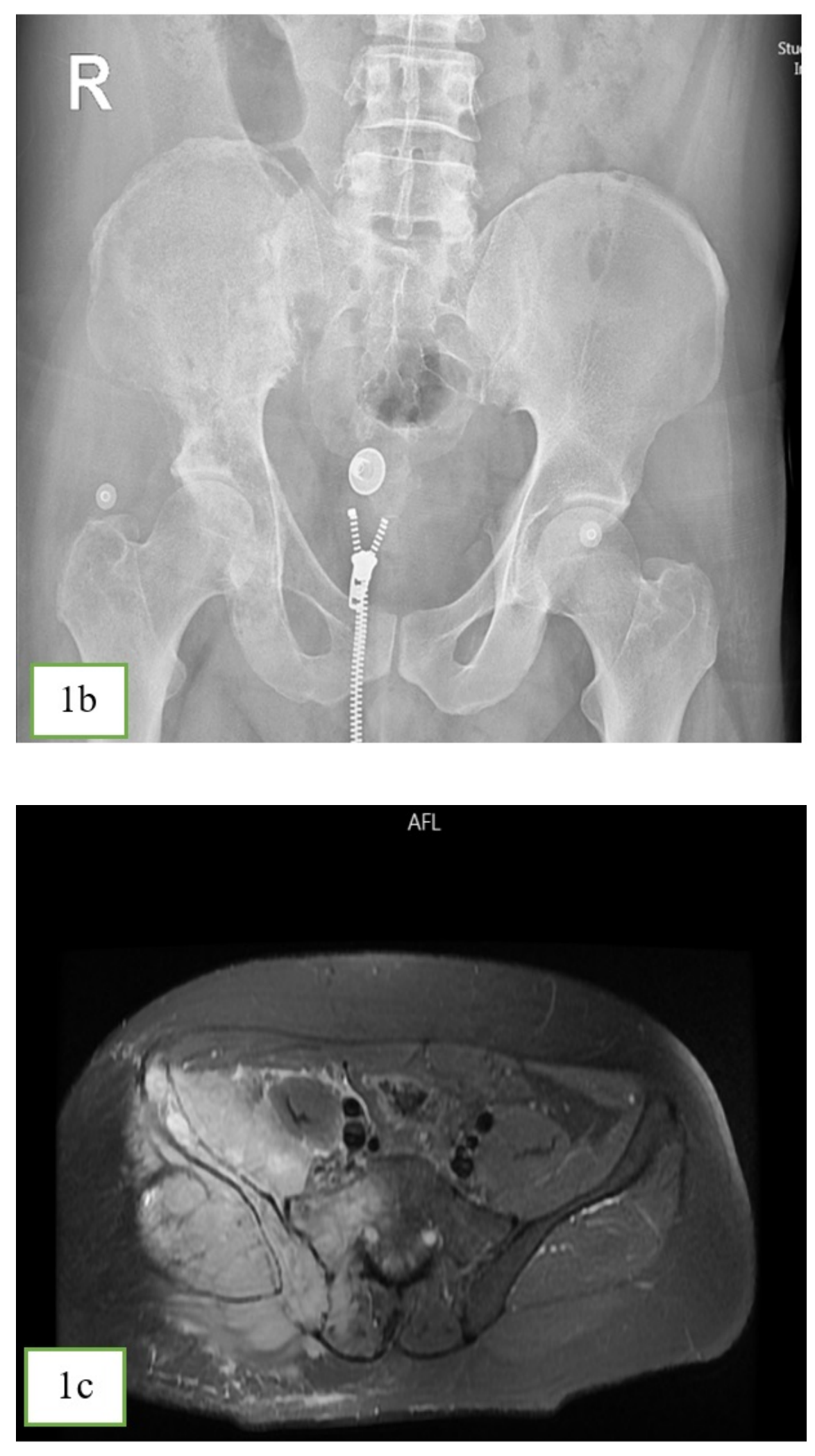


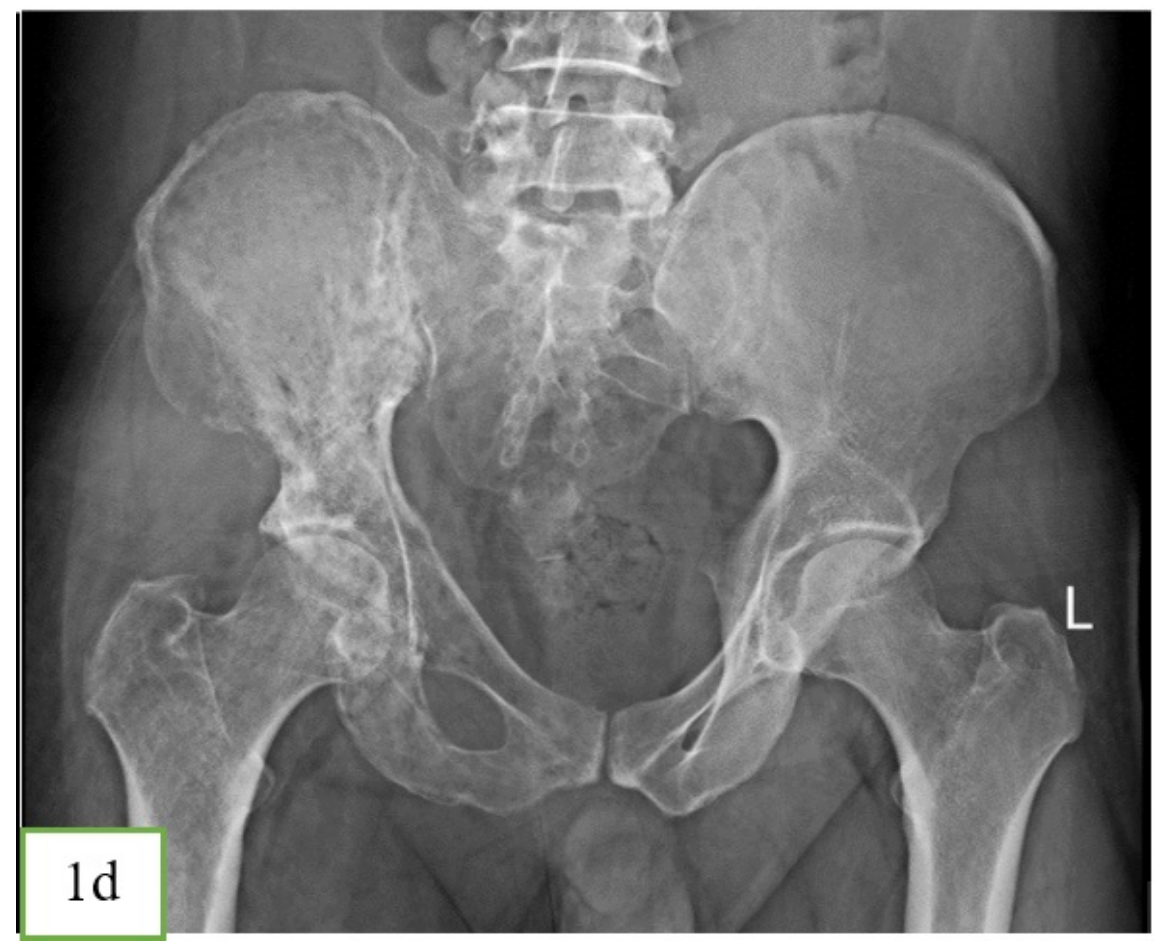

Figure 1: b \&d: X-ray images of the pelvis that demonstrate lytic radiolucent lesion with destructive pattern and sclerotic areas, (image d taken 4 months later from b ), a\& c T2-weighted images with fat saturation, showing high signal intensity in the right iliac and superior ramus of pubis, abnormal high signal intensity in the medulla of femur and sequestration in iliac bone is also seen.

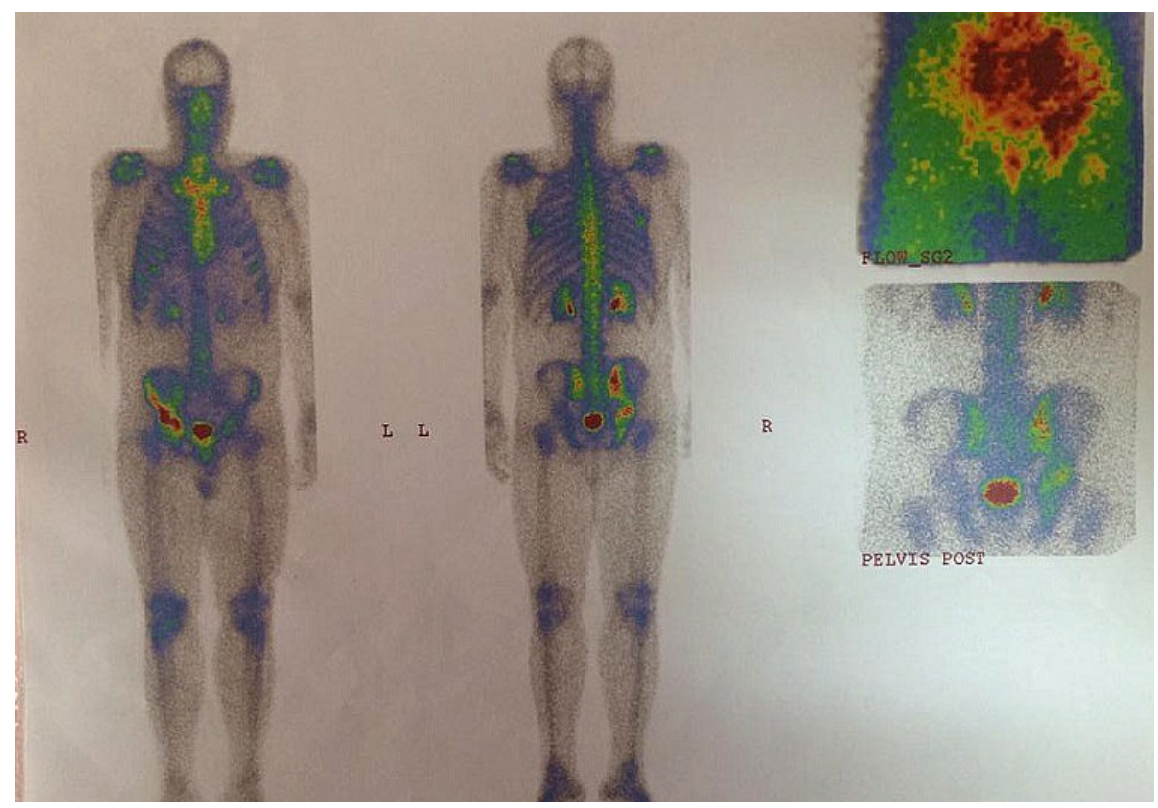

Figure 2: the scan showed moderately to severely increased radiotracer uptake in the right sacroiliac joint, right acetabular joint, and iliac crest. 

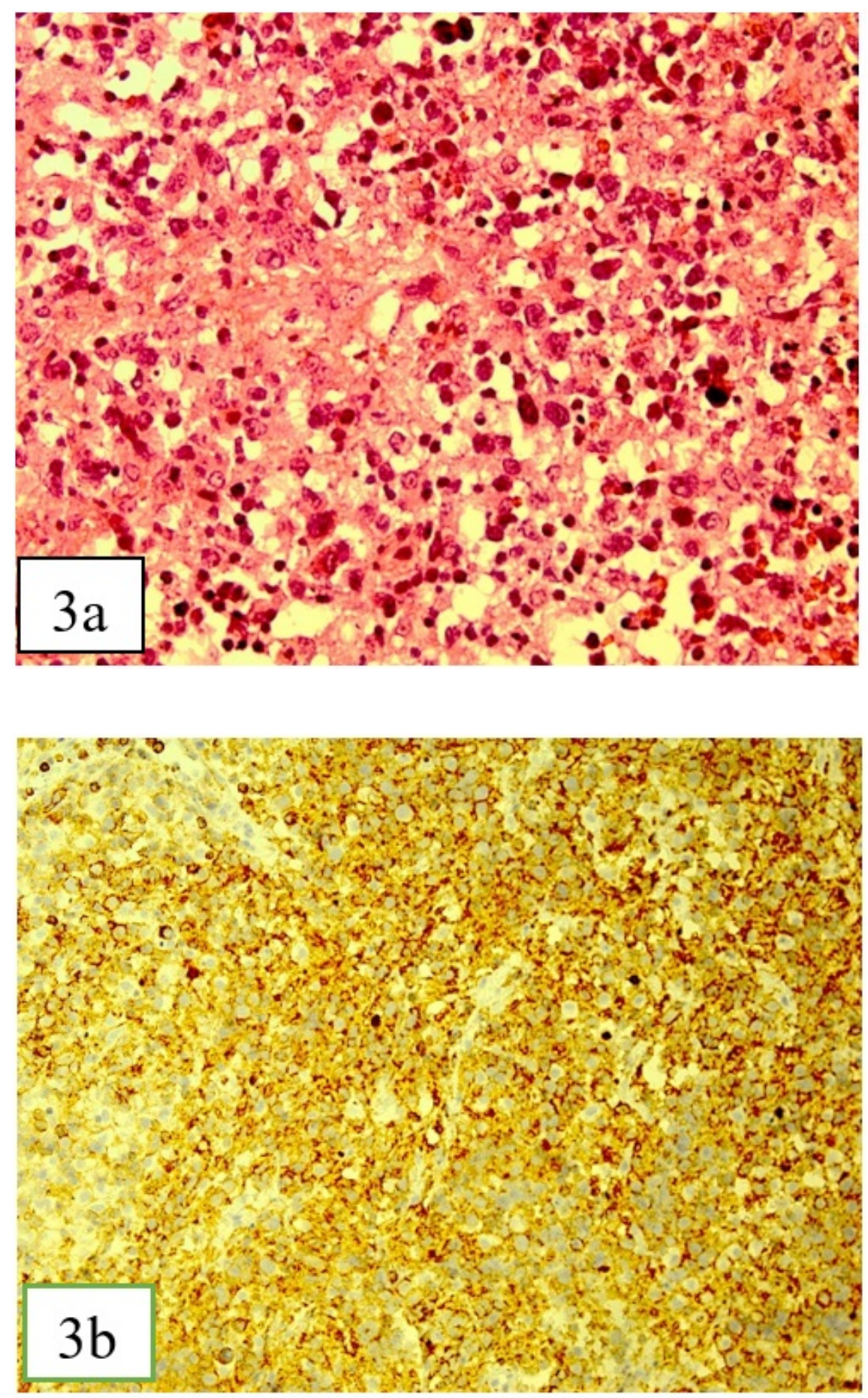

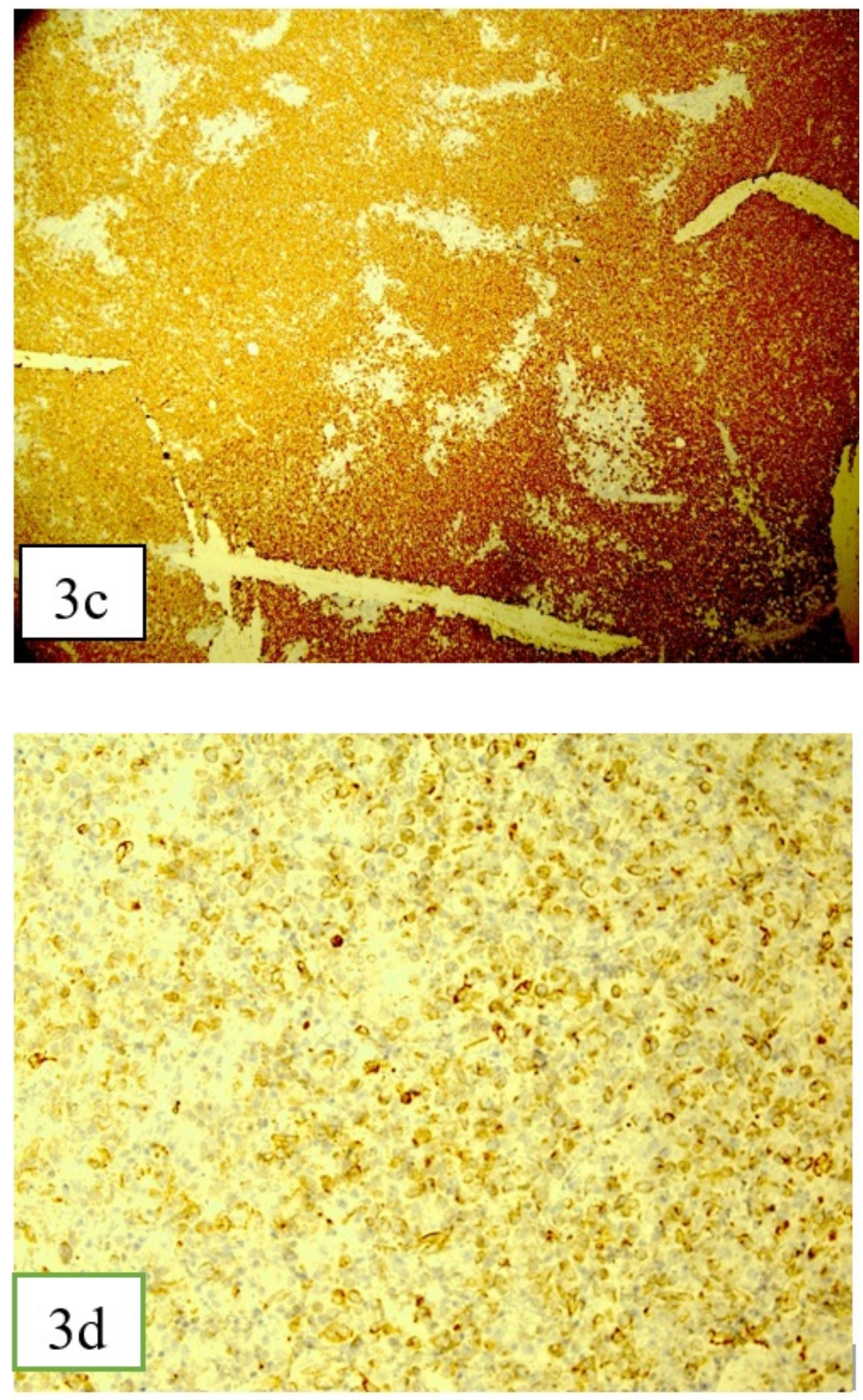

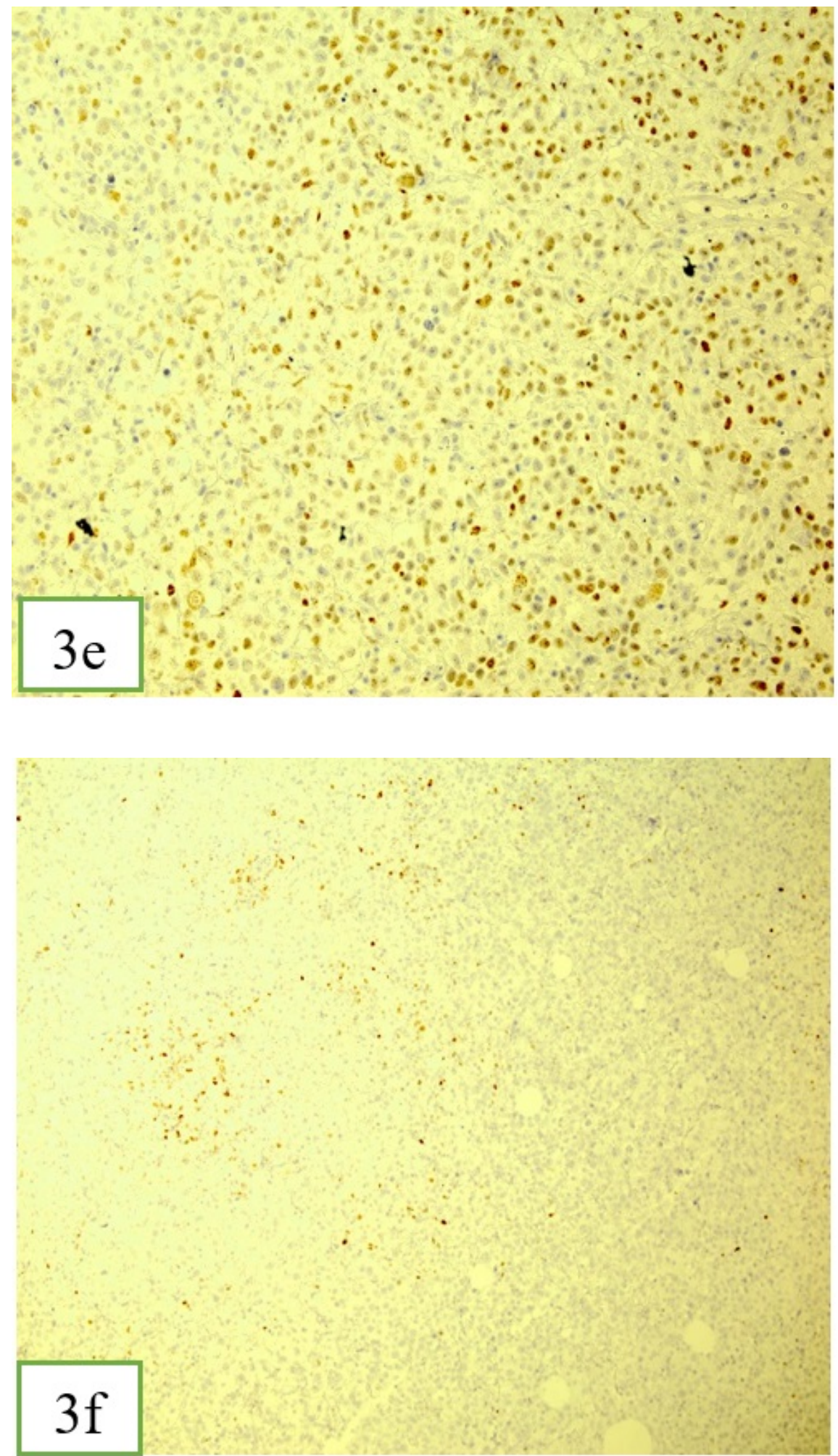

Figure 3: Pathology and IHC of primary bone diffuse large B-cell lymphoma, non-GCB, (A ) H\&E, (B ) LCA, (C) CD20 (D ) bcl2 (E ) bcl6 ,( F) MUM-1+, 

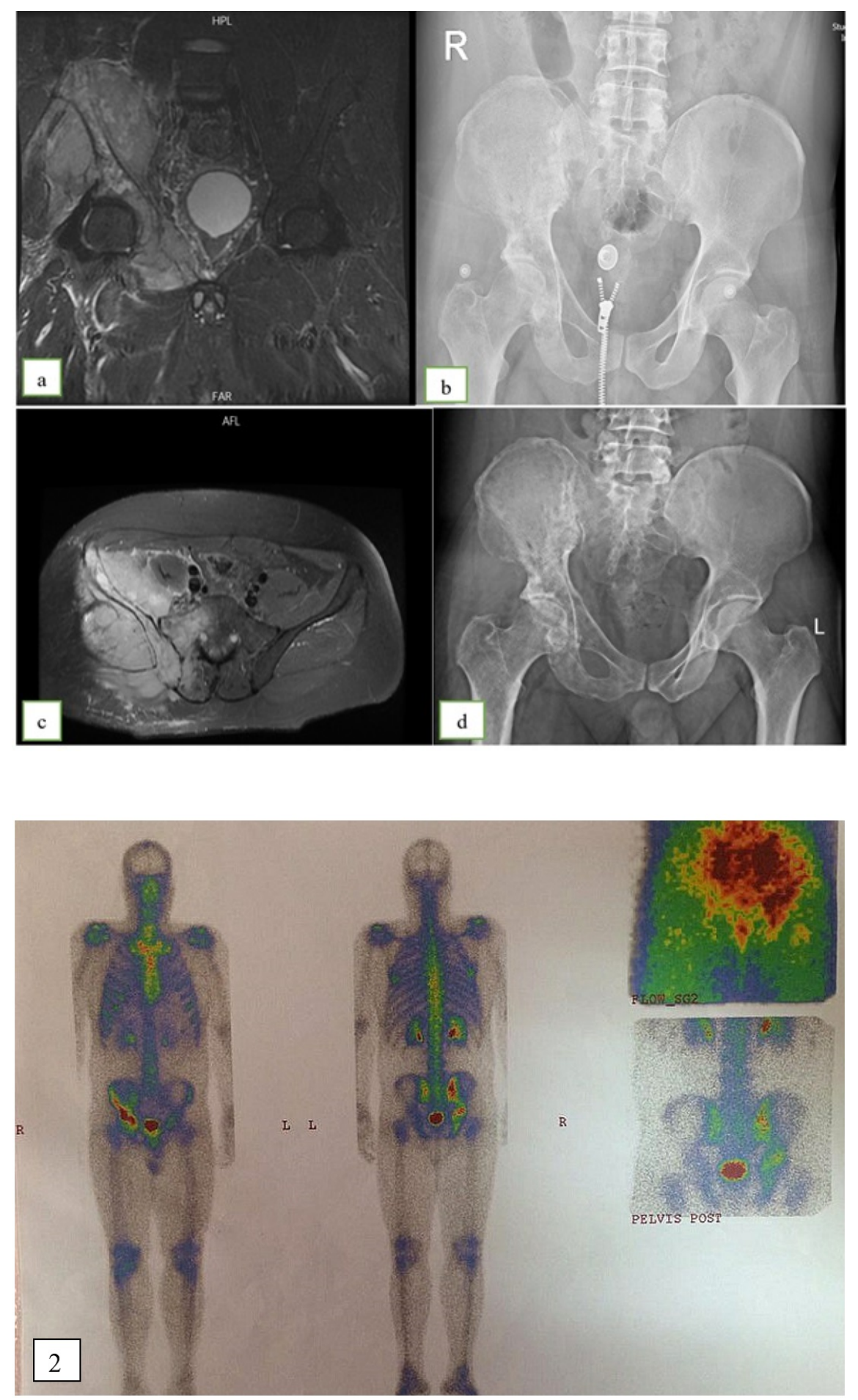

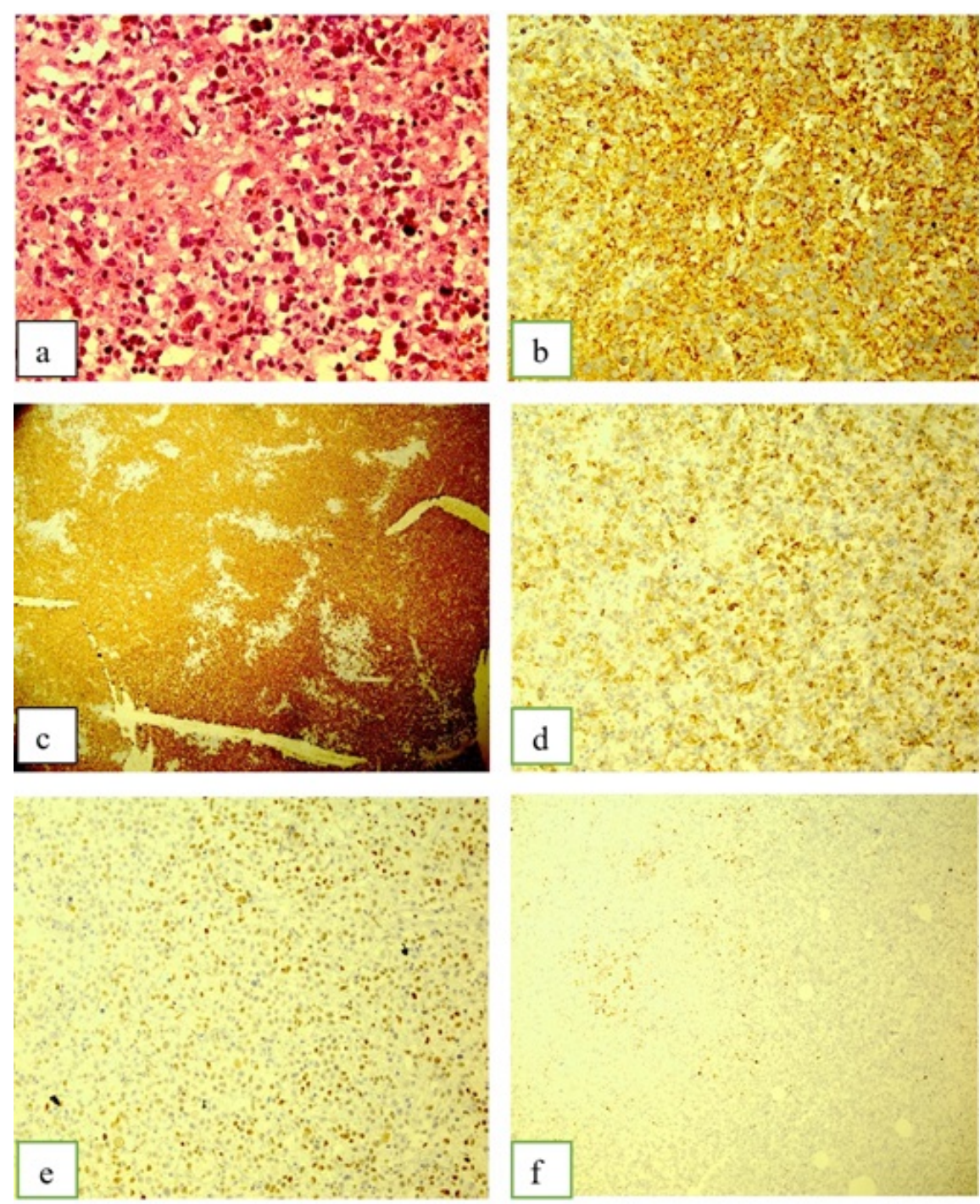